\title{
Influence of Teeth Clenching on the Bodily Equilibrium against Striking Weight Impact
}

\author{
Shinsuke Tanaka, DDS, PhD, Toshihiro Hirai, DDS, PhD, Hisashi Koshino, DDS, PhD, \\ Yuichi Yokoyama, DDS, PhD, and Tsutomu Ishijima, DDS, PhD
}

Department of Removable Prosthodontics, School of Dentistry, Health Sciences University of Hokkaido, Ishikari-Tobetsu, Hokkaido, Japan

\section{Clinical significance}

The results of this study suggested that teeth clenching stabilizes the head in cooperation with the activity of the sternocleidomastoid muscles, and prevents head movements and body sway during striking weight impact. It was speculated that teeth clenching might play an effective role in the enhancement of sports performance.

\begin{abstract}
Purpose: The purpose of this study was to elucidate the influence of teeth clenching on the head posture and body sway during an impact lord to the greater trochanter of the femur.

Methods: The sternocleidomastoid muscle activities, head posture, and the total locus length of head movement and body sway during and in the absence of a striking weight impact under the resting position of the mandible and during $50 \%$ and $100 \%$ voluntary maximal clenching were analyzed.
\end{abstract}

Results: 1) The activity of the sternocleidomastoid muscles increased with the teeth clenching force, and the increase was greater during a striking weight impact than that in the absence of a striking weight impact. 2) The decrease in the lateral bending and rotation angles of the neck observed during a striking weight impact was greater under the voluntary teeth clenching conditions as compared with that under the resting position of the mandible. 3) The total locus length of the head movement and body sway were shorter under the voluntary teeth clenching conditions as compared with that under the resting position of the mandible. 4) In contrast to the observations under the resting position of the mandible during a striking weight impact, the ratio of the total locus length of the head movement to that of the body sway under the voluntary teeth clenching during a striking weight im-

\section{Corresponding to: Dr Shinsuke Tanaka}

Department of Removable Prosthodontics,

School of Dentistry, Health Sciences University of Hokkaido, 1757 Kanazawa, Ishikari-Tobetsu, Hokkaido 061-02, Japan

Tel \& Fax: +81-133-23-1211

E-mail: shinco@hoku-iryo-u.ac.jp

Received on January 6, 2006 / Accepted on May 23, 2006 pact decreased to the level noted under the resting position of the mandible in the absence of a striking weight impact.

Conclusion: The results of this study suggest that teeth clenching stabilizes the head in cooperation with the activities of the sternocleidomastoid muscles, and prevents excessive head movement and body sway during a striking weight impact.

Key words: teeth clenching, sternocleidomastoid muscle, head posture, head movement, body sway

\section{Introduction}

In a study conducted previously, involuntary clenching at the intercuspul position was observed during physical exertion in 58 percent of subjects with normal dentition. ${ }^{1}$ The elbow flexion strength, back strength, grip strength, and the anaerobic power were shown to be greater with the mandibles at the intercuspal position (centric occlusion) than in eccentric mandibular positions in subjects who exhibited powerful clenching during exercise. ${ }^{2,3}$ We also reported that the reaction time as an index of quickness was significantly prolonged when the subjects lost their occlusal support. ${ }^{4}$ These results suggest that a close relationship exists between dental occlusion and physical exercise.

It has been reported that the sternocleidomastoid muscles are active during occlusal activity., It has also been reported that occlusal treatments are effective for the relief of sternocleidomastoid muscle pain. ${ }^{7-9}$ We reported that there was a close relationship between occlusion and head posture, and suggested that an imbalance in occlusal support might cause an imbalance in the activities of the sternocleidomastoid muscles and head posture. ${ }^{10}$

The purpose of this study was to elucidate the influence of teeth clenching on the head posture 


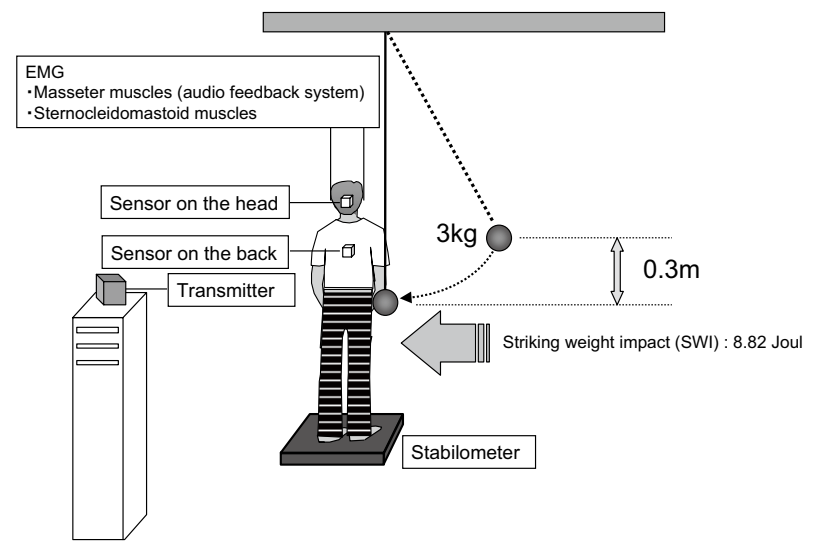

Fig. 1 Set up of the experiment.

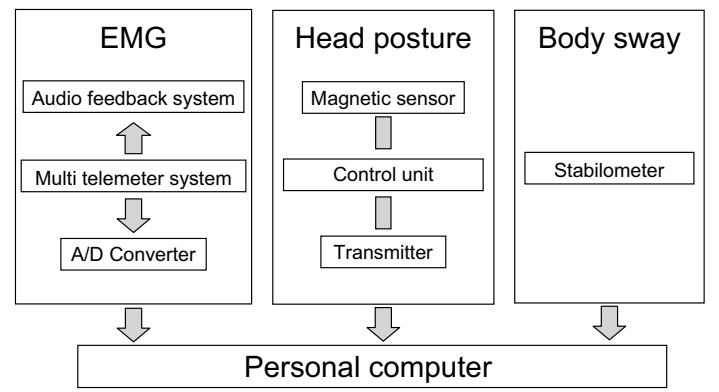

Fig. 2 Block diagram of the measuring system.

and body sway during application of an impact load to the greater trochanter of the femur.

\section{Materials and methods}

Five male subjects not suffering from any apparent clinical abnormalities of the stomatognathic and auditory-equilibrium systems were selected from among the students and faculty of our university (average age: $27.4 \pm 3.4$ years). Informed consent was obtained from each of the subjects prior to their participation in the experiments.

A $3-\mathrm{kg}$ sandbag was placed at the end of a rope which the length was adjusted for each subject, and placed so as to strike the subjects on the right greater trochanter of the femur while they stood in the upright position on the stabilometer under the eyes-closed condition, after 20 seconds of eye closure (Fig. 1 and 2). The loading direction of the impact was set to be perpendicular to the sagittal plane of the trunk. The striking weight impact (hereafter referred to as SWI) was set so that the subjects did not feel any unpleasant sensation, such as pain, and could remain on the stabilometer during the measurements. In this study, the impact strength was set to be 8.8J, using a $3-\mathrm{kg}$ sandbag on a pendulum from a

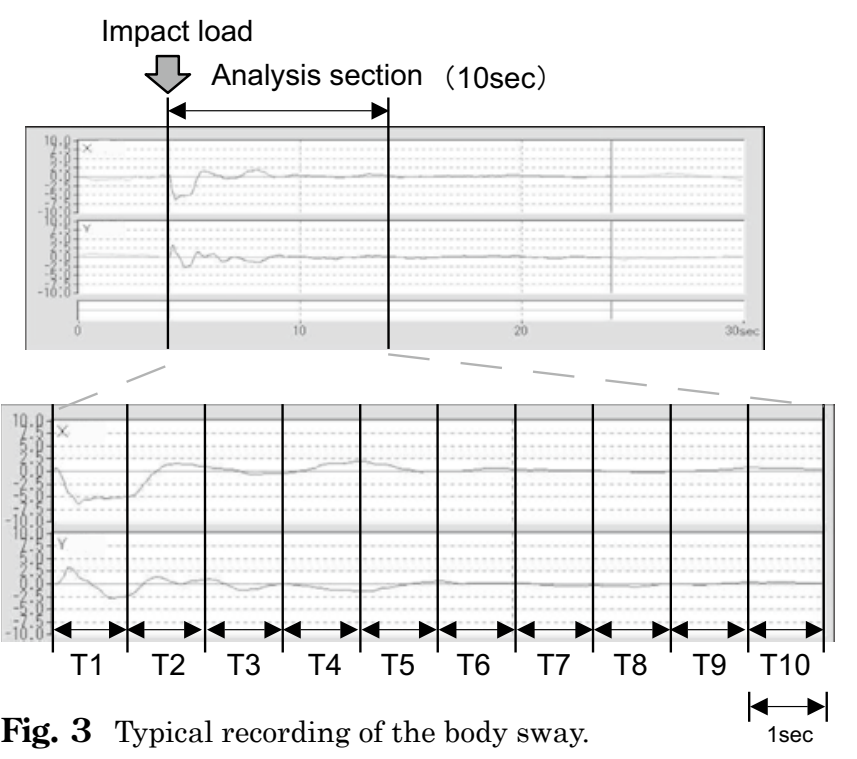

height of 30 centimeters.

Each subject was directed to keep the mandibular position at rest (hereafter referred to as REST) and at the intercuspal position with 50\% and $100 \%$ voluntary maximal clenching (hereafter referred to as 50\% VMC and 100\% VMC, respectively) for 10 seconds each. Each VMC condition was controlled by an audio feedback system (MIYUKI GIKEN Co.) to measure the right and left masseter muscle activities. The audio feedback system evaluated the muscle activities of the right and left masseter muscles, and the electric sound of the instrument disappeared within $\pm 5 \%$ of the goal value.

The head movement, body sway and electromyographic (EMG) activity were recorded at REST and during 50\% and 100\% VMC, during and in the absence of the SWI. Measurements were performed 3 times under each condition with 5-minutes' rest intervals. For the analyses of the head movement and body sway, the 10- sec- $^{-}$ onds' recording during the SWI was divided into 10 sections (T1 - T10) (Fig. 3).

The head posture and head movements were recorded by an electromagnetic, six-degree-offreedom tracking instrument (POLHEMUS Co. 3 SPACE-WIN), and the body sway was recorded by the stabilometer (NEC Medical Systems Co. EB1101).

The instrument for measuring the head posture and head movements indicated the movements of the sensor by three-dimensional coordinates and orientation angles to each axis of the coordinates. One sensor was fixed at the back of the subject at the level of the 5th - 8th thoracic 


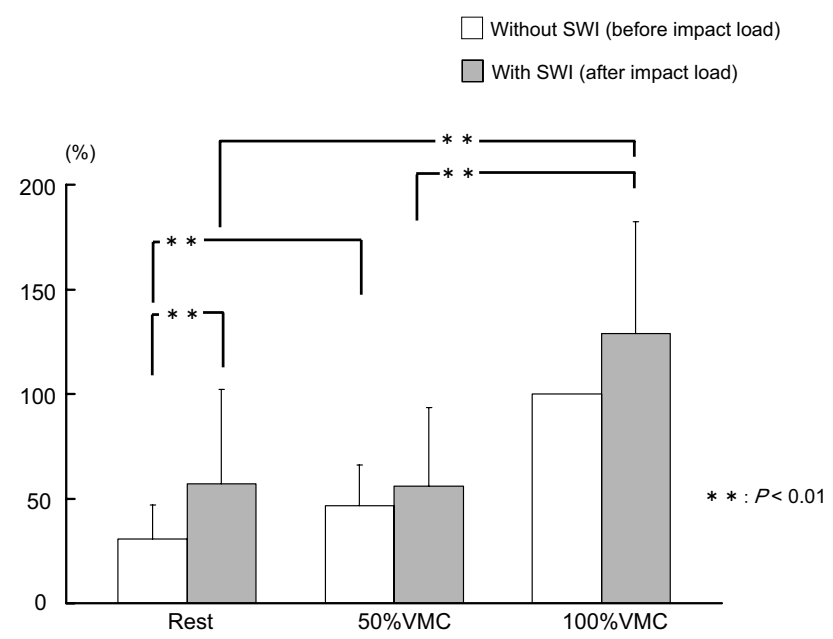

Fig. 4 Sternocleidomastoid muscle activity during and in the absence of the SWI (relative muscle activity).

vertebra using surgical tape and the other sensor was fixed on the back of the head with a belt. The relative angle and positions of the two sensors were measured. The distance from the transmitter to the sensor was set to be within $76 \mathrm{~cm}$, according as instruction for use. During the measurements, magnetic metals and personal computers were removed from the measuring area.

The stabilometer indicated the movements of the center of the foot in the upright standing position on the stabilometer. The head movements and body sway were evaluated by the total locus length of each. The EMG activity of the masseter muscles and the sternocleidomastoid muscles were recorded simultaneously. The EMG activities were recorded from the masseter muscles and middle part of the sternocleidomastoid muscles of either side. Bipolar Ag - AgCl surface electrodes with an inter-electrode distance of $20 \mathrm{~mm}$ were used for the measurements. The electrodes were placed by palpation of the muscles along the main direction of the muscle fibers. The time constant was 0.01 seconds. The four EMG signals were stored in a personal computer through an A/D converter (sampling rate $1200 \mathrm{~Hz}$ ). Statistical analysis was performed by one-way analysis of variance(ANOVA), Scheffe's test $(P<0.05)$, and regression analysis (SPSS for Windows 9.0, SPSS Inc.).

\section{Results}

The EMG activities of the sternocleidomastoid muscles (relative muscle activity) in the absence

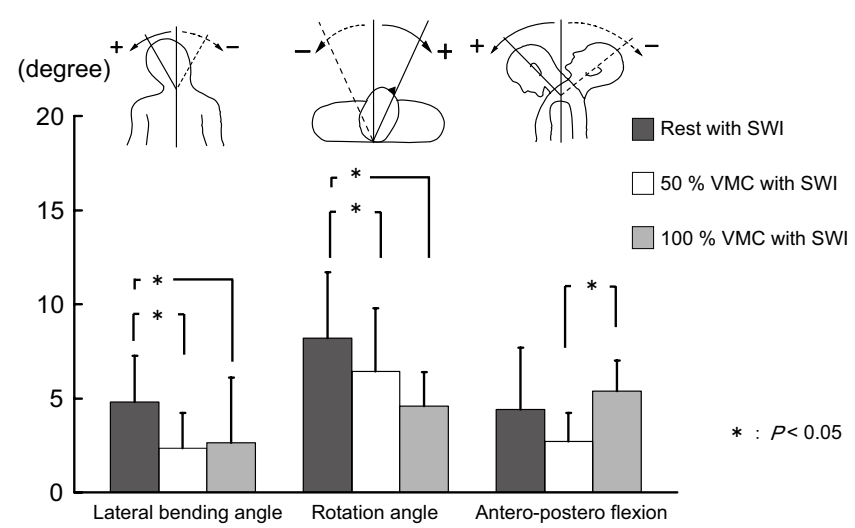

Fig. 5 Lateral bending angle, rotation angle, and anteroposterior flexion angle of the neck during the SWI at REST and during 50\% and 100\% VMC.

of the SWI were $30.8 \pm 16.3 \%$ at REST and $46.5 \pm$ $19.6 \%$ with $50 \%$ VMC, when the value recorded with $100 \%$ VMC was taken as $100 \%$ (Fig. 4). The EMG activities of the sternocleidomastoid muscles with $100 \%$ VMC in the absence of the SWI were statistically significantly greater $(P<0.01)$ than those at REST and with 50\% VMC, and the muscle activities with $50 \%$ VMC were statistically significantly greater $(P<0.01)$ than those at REST (Fig. 4).

On the other hand, the EMG activities of the sternocleidomastoid muscles during the SWI were $57.2 \pm 44.8 \%$ at REST, $56.3 \pm 37.3 \%$ with $50 \%$ VMC, and $129.0 \pm 53.5 \%$ with $100 \%$ VMC, when the value with $100 \% \mathrm{VMC}$ in the absence of the SWI was taken as $100 \%$ (Fig. 4). The EMG activities of the sternocleidomastoid muscles with 100 $\%$ VMC were thus statistically significantly greater than those at REST and with $50 \%$ VMC during the SWI $(P<0.01)$ (Fig. 4).

The maximal swing values of the lateral bending angle of the neck during the SWI were $4.8^{\circ} \pm$ $3.5^{\circ}$ at REST, $2.4^{\circ} \pm 1.3^{\circ}$ with $50 \% \mathrm{VMC}$, and $2.6^{\circ} \pm$ $1.6^{\circ}$ with $100 \% \mathrm{VMC}$ (Fig. 5). The lateral bending angles of the neck during the SWI with $50 \%$ VMC and $100 \%$ VMC were thus statistically significantly smaller than those at REST $(P<0.05)$ (Fig. 5).

The maximal swing values of the rotation angle of the neck during the SWI were $5.6^{\circ} \pm 2.7^{\circ}$ at REST, $3.9^{\circ} \pm 2.0^{\circ}$ with $0 \% \mathrm{VMC}$ and $3.6^{\circ} \pm 1.9^{\circ}$ with $100 \%$ VMC (Fig. 5). The rotation angles of the neck during the SWI with 50\% VMC and 100\% VMC were statistically significantly smaller than the angle at REST $(P<0.05)$ (Fig. 5).

The maximal swing values of the antero-pos- 

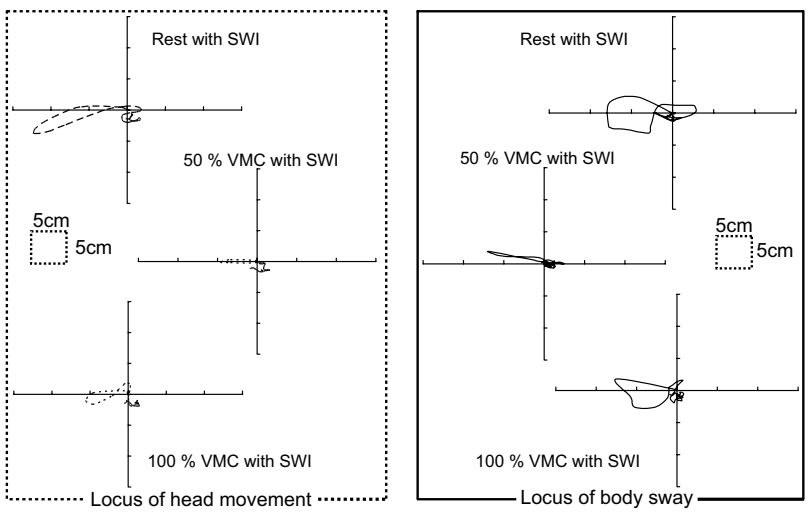

Fig. 6 Typical recording of the total locus length of the head movement and body sway during the SWI at REST and during 50\% and 100\% VMC.
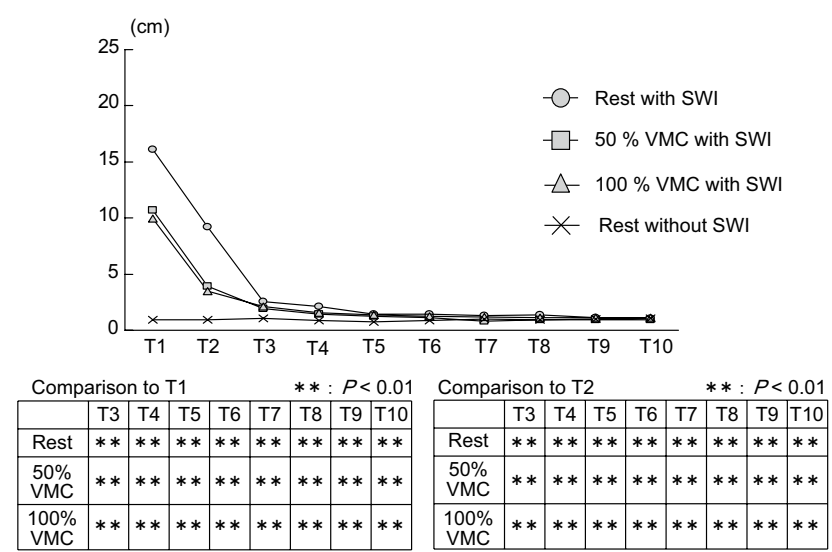

Fig. 7 Time-course of changes of the total locus length of the head movement and statistical analysis.

tero flexion angle of the neck during the SWI were $4.4^{\circ} \pm 2.8^{\circ}$ at REST, $2.7^{\circ} \pm 1.2^{\circ}$ with $50 \%$ VMC and $5.4^{\circ} \pm 2.7^{\circ}$ with $100 \%$ VMC (Fig. 5). The antero-postero flexion angles of the neck during the SWI with $50 \%$ VMC was statistically smaller than that with $100 \%$ VMC $(P<0.05)$ (Fig. 5).

The typical patterns of the total locus length of the head movement and the body sway at REST, and with $50 \%$ and $100 \%$ VMC during the SWI of a subject are shown in Figure 6. The findings showed that the total locus lengths of the head movement and body sway with $50 \%$ and $100 \%$ VMC were shorter than the value at REST. Similar findings were recorded in all of the subjects.

The total locus length of the head movements and the body sway at REST, and with $50 \%$ and $100 \%$ VMC during the SWI in the T1 and T2 sections were statistically significantly longer than the corresponding values in the T3 to T10 sections $(P<0.01)$ (Figs. 7,8$)$. In the T2 section, the total locus length of the head movement and the body sway with 50\% VMC and 100\% VMC were
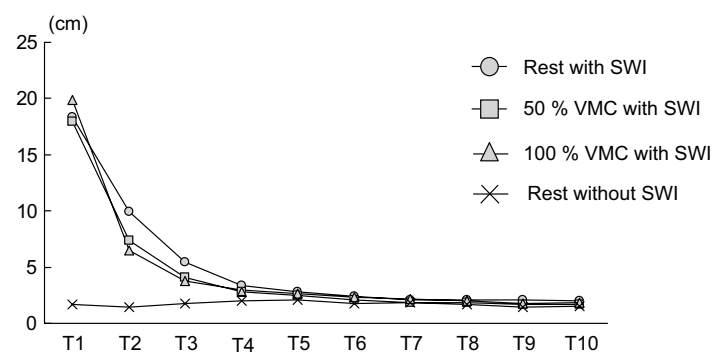

\begin{tabular}{|c|c|c|c|c|c|c|c|c|c|c|c|c|c|c|c|c|c|}
\hline \multicolumn{6}{|c|}{ Comparison to $\mathrm{T} 1$} & $* *$ & \multicolumn{2}{|c|}{$: P<0.01$} & \multicolumn{6}{|c|}{ Comparison to $\mathrm{T} 2$} & $* *$ & \multicolumn{2}{|c|}{$P<0.01$} \\
\hline & T3 & T4 & T5 & T6 & T7 & T8 & T9 & T10 & & T3 & T4 & T5 & T6 & \begin{tabular}{|l} 
T7 \\
\end{tabular} & T8 & T9 & T10 \\
\hline Rest & ** & ** & ** & ** & ** & ** & ** & ** & Rest & $* *$ & $* *$ & $* *$ & $* *$ & $* *$ & $* *$ & $* *$ & ** \\
\hline $\begin{array}{l}50 \% \\
\text { VMC }\end{array}$ & ** & $* *$ & ** & $* *$ & $* *$ & $* *$ & $* *$ & $* *$ & $\begin{array}{l}50 \% \\
\text { VMC }\end{array}$ & $* *$ & $* *$ & $* *$ & $* *$ & $* *$ & $* *$ & $* *$ & $* *$ \\
\hline $\begin{array}{l}100 \% \\
\text { VMC }\end{array}$ & $* *$ & $* *$ & ** & ** & ** & ** & $* *$ & $* *$ & $\begin{array}{l}100 \% \\
\text { VMC }\end{array}$ & $* *$ & $* *$ & ** & $* *$ & $* *$ & ** & $* *$ & ** \\
\hline
\end{tabular}

Fig. 8 Time-course of changes of the total locus length of body sway and statistical analysis.

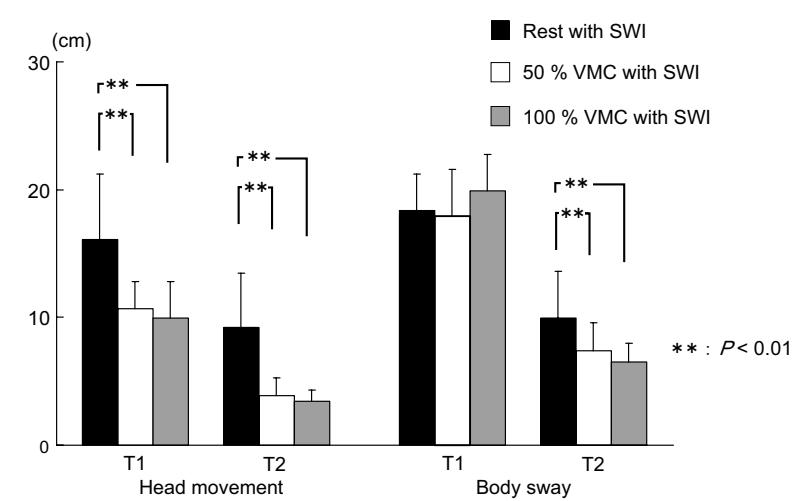

Fig. 9 Total locus lengths of the head movement and body sway in the T1 and T2 sections.

statistically significantly shorter than that at $\operatorname{REST}(P<0.01)$ (Fig. 9).

A positive correlation was found between the total locus length of the head movement and that of the body sway during the SWI at REST $\left(r^{2}=0.29, P<0.01\right)$ and with $50 \% \operatorname{VMC}\left(r^{2}=0.57\right.$, $P<0.05)$ in the T2 section (Fig. 10). However, no such positive correlation was found in the T2 section with 100\% VMC (Fig. 10).

The ratio of the locus length of the head movement: that of the body sway in the T2 section during the SWI was $0.9 \pm 0.3$ at REST, $0.6 \pm 0.2$ with $50 \% \mathrm{VMC}$, and $0.6 \pm 0.2$ with $100 \%$ VMC. The ratios with $50 \%$ VMC and $100 \%$ VMC were statistically significantly smaller as compared to the ratio at REST during the SWI, $(P<0.01)$, and were similar to those at REST without the SWI (Fig. 11). 


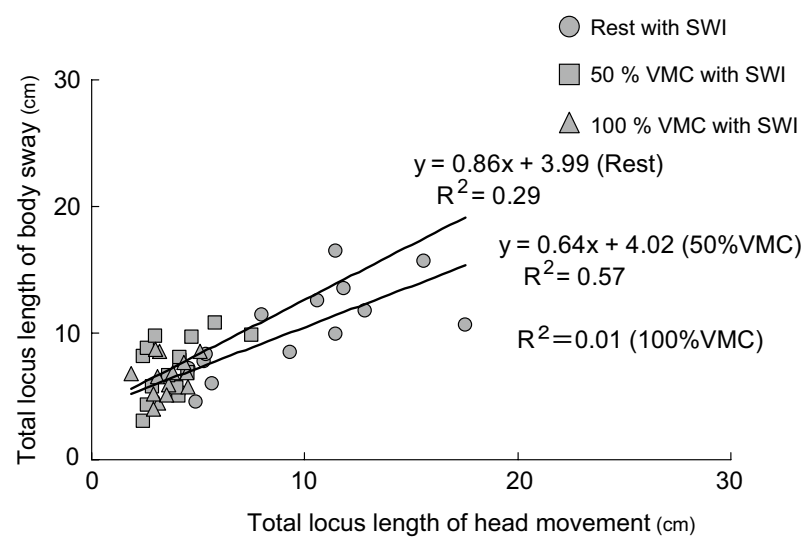

Fig. 10 Relationship between the total locus length of the head movement and body sway in the T2 section.

\section{Discussion}

Under the hypothesis that teeth clenching plays a role in the fixation of the head and contributes to the prevention of head movements and body sway during the application of an impact load to the greater trochanter of the femur, the relationships among the clenching force, sternocleidomastoid muscle activity, head position, head movement and body sway were all analyzed and discussed further.

Because the sense of equilibrium is related to vestibular sensations, visual sensations and somatic sensations, the experiments in the study were performed under the eyes-closed condition, after 20 seconds of eye closure. As to the impact load applied on the subjects, a load of 8.8 Joules, which could disturb the body balance of the subjects, was applied to the greater trochanter of the femur; however, the subjects could remain standing on the stabilometer and did not complain of any pain or discomfort. The SWI was set within a range where the subjects could remain standing on the stabilromter during the measurement without feeling any discomfort. The target of the SWI was set to be the greater trochanter of the femur on the assumption that the center of gravity line is the index of body balance.

It has been reported that the activities of the sternocleidomastoid muscles are synchronized to those of the jaw closing muscles during mastication and involuntary jaw clenching. ${ }^{5,11-13}$ The increases of the sternocleidomastoid muscle activities with increase of the jaw clenching force during and in the absence of the SWI in the present study were consistent with these previous reports. The increase of the sternocleidomastoid

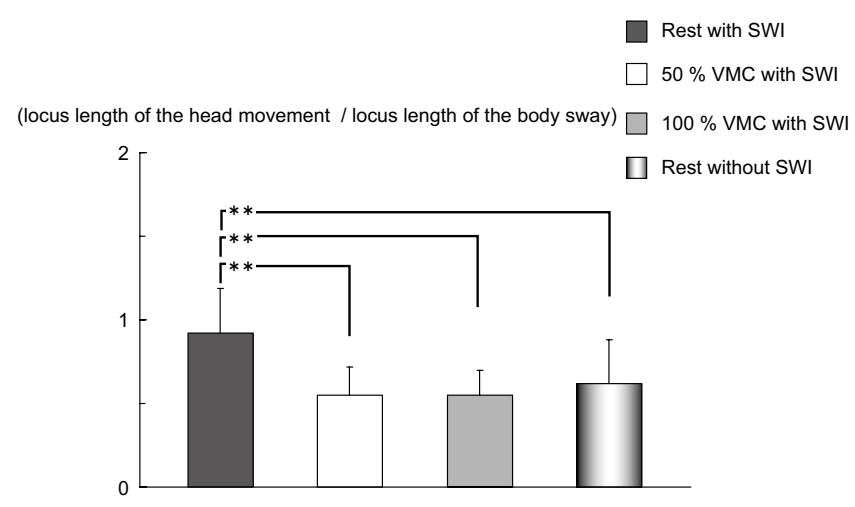

Fig. 11 Ratio of the locus length of the head movement to that of the body sway in the T2 section.

muscle activities, decrease of the lateral bending angles and/or rotation angles of the neck, and reduction of the total locus length of the head movements during the SWI under the teeth clenching conditions (50\% VMI and 100\% VMI) as compared with that in the non-clenched condition (REST) in the present study are considered to indicate that the sternocleidomastoid muscles participate in head fixation in cooperation with the jaw closing muscles.

A close correlation has been recognized between trigeminal inputs and the activities of the neck muscles, ${ }^{14}$ which has been referred to as the trigemino-neck reflex. ${ }^{15,16}$ Moreover, it has been reported by Luo et al, based on the results of electrophysiological and neuroanatomical studies, that the neck muscles participate with the trigeminal nerve. ${ }^{17,18}$ They suggest that sensory feedback from the masticatory muscles is subjected to presynaptic inhibition and integration prior to reaching the thalamus. The projection of jaw-muscle spindle afferents to caudal brainstem regions may play a significant role in masticatory-muscle stretch reflexes and in the integration of trigeminal proprioceptive information and its transmission to higher centers. It is speculated that the activities of the muscle spindles and mechanoreceptors of the periodontal ligament influence the activities of the motor neuron pool of the sternocleidomastoid muscles through the central ramus of the mesencephalic trigeminal neuron. ${ }^{19}$ Teeth clenching is speculated to contribute to fixation of the head during SWI, in $\mathrm{co}^{-}$ operation with sternocleidomastoid muscle activity.

The reduction of the total locus length of the body sway during the SWI may be attributable to the reduction of the total locus length of the head movement. The total locus length indicating the 
magnitude of the body movement is considered to be useful for analysis of the relationship between the head movement and body sway. The standing posture of human beings is often physically unstable, because of the high center of gravity and the presence of the heavy cranium positioned on top of the vertebral column. ${ }^{20}$ Consequently, it would be reasonable to assume that changes of the head posture at the time of standing would have an influence on the sway of the entire body.

Due to the close relationship between the total locus length of head movement and that of the body sway, we examined the ratio of the locus length of head movement to that of body sway as a parameter of body balance. The ratio in the $a b^{-}$ sence of a SWI, with the subject just standing on the stabilometer under REST, is considered to indicate that in the presence of an adequate body balance. Our observation in this study of a higher ratio at REST during the SWI than at REST in the absence of the SWI indicates that the body balance was disturbed by the SWI. As shown in the results, the ratio under the teeth clenching conditions during the SWI was similar in values to the value at REST in the absence of the SWI. This result appears to suggest that teeth clenching prevents head movements and body sway. A higher antero-postero flexion angle of the neck was noted with $100 \%$ VMC as compared to that with 50\% VMC during the SWI. It is speculated that clenching force above a certain level causes excessive forward neck bending. Taking into consideration the observation that the antero-postero flexion angle of the neck with 100\% VMC was larger than that with $50 \%$ VMC during the SWI, resulting in an increase of the body sway, $50 \% \mathrm{VMC}$ is speculated to be a more suitable clenching force to prevent head movements during the application of SWI, as compared to $100 \%$ VMC. The results of this study suggest excessive muscle activity of the cervical muscles can change the head position. Kibana has reported that the head bends forward with voluntary maximal clenching. ${ }^{10}$ The total locus length of the body sway was higher with $100 \%$ VMC as compared to that with $50 \% \mathrm{VMC}$, differing from the results for the total locus length of the head movements in this experiment. It is speculated that anterior flexion of the neck caused by the activity of the cervical muscles during teeth clenching was increased by the excessive clenching force resulting in the increase in the locus length of the body sway.
It has been demonstrated that even simple and small movements of a local body part can produce a component pattern of muscular activity, involving not only the muscles directly producing the observed movement, but also other muscles often located remotely the moving part. ${ }^{21}$ The relationship between the activities of the sternocleidomastoid muscles and the jaw closing muscles were described in the above study.

Fixation of the head is supposed to contribute to stabilization of eyes and protection from head injury. In addition to fixation of the head, control of body sway seems to be essential for best performance in sports activities. Teeth clenching has now been shown to play an important role in head movements during SWI.

\section{Conclusion}

The existence of an interdependent relationship between stomatognathic functions, especially the occlusal function, and body equilibrium was confirmed. The results of this study suggest that $50 \%$ VMC plays an important role in fixation of the head in cooperation with the sternocleidomastoid muscle activities, and prevents excessive head movements and body sway during a SWI. It is also speculated based on the study results that teeth clenching might play an effective role in the enhancement of sports performance.

\section{References}

1. Ishijima T, Hirai T, Imamura $\mathrm{T}$ et al. The frequency of clenching during physical exertion. J Jpn Prosthodont Soc 35: 193-199, 1991.

2. Ishijima T, Ichioka N, Konishi Y et al. Mandibular position and physical exercise. In: Tsuru H, Preiskel HW, Matsuo E et al, editors, Advanced prosthdontics worldwide 530-531, Hiroshima: WCP Publication Committee, 1991.

3. Ichioka N. Correlation of motor functions between jaw and body -elbow flexion strength during teeth clenching in horizontally different mandibular positions -. J Jpn Prosthodont Soc 39: 213-224, 1995.

4. Ishijima T, Hirai T, Koshino $\mathrm{H}$ et al. The relationship between occlusal support and physical exercise ability. J Oral Rehabil 25: 468-471, 1998.

5. Davis PL. Electromyographic study of superficial neck muscle in mandiblar function. J Dent Res 58: 537-538, 1979.

6. Kawamura T. Electromyographic study on the activities of neck muscles during various mandibular 
movements. Bull Tokyo Med Dent Univ 50: 94-115, 1983.

7. Gelb H, Calderone JP, Gross SM et al. The role of the dentist and the otolaryngologist in evaluating temporomandibular joint syndromes. J Prosthet Dent 18: 497-503, 1967.

8. Kohno S, Miura H, Yoshida K et al. A pain in the sternocleidomastoid muscle and a defect in the anterior guidance. Proc J Jpn Soc. Stomatognath Funct 3: 195-198, 1985.

9. Kohno S, Kobayashi H, Yoshida K. Pain in The sternocleidomastoid muscle and defects of anterior guidance. J Jpn Prosthodont Soc 31: 409-417, 1987.

10. Kibana Y, Ishijima T, Hirai T. Occlusal support and head posture. J Oral Rehabil 29: 58-63, 2002.

11. Kohno S, Yoshida K, Kobayashi $\mathrm{H}$ et al. The EMG Activities of the Sternocleidomastoid Muscle during Occlusal Function. J Jpn Prosthodont Soc 31: 764-769, 1987.

12. Yoshida K. Electromyographical Analysis of function of sternocleidomastoid muscle during occlusal function. Bull Tokyo Med Dent Univ 55: 53-70, 1988.

13. Nagasuna T, Tanaka M, Kawazoe T et al. Postural synergy of sternocleidomastoid muscle for control of head position during ballistic voluntary jaw movements. J Jpn Prosthodont Soc 37: 1232-1237, 1993.

14. Manni E, Palmeieri G, Marini R et al. Trigeminal influence on extensor muscles of the neck. Exp Neurol 47: 330-342, 1975.

15. Sumino R, Nozaki S, Kahoh M. Trigemino-neck reflex. In: Kawamura Y, Dubner R, editors, Oro-facial sensory and motor function 81-88, Tokyo: Quintessence, 1981.

16. Sumino R, Nozaki S. Trigemino-neck reflex. In: Anderson DJ, Matthews B, editors, Its peripheral and central organization: In Pain in trigeminal region 365-374, New York: North Holland Biomedical Press, 1997.

17. Luo P, Wong R, Dessem D. Projection of jaw-muscle spindle afferents to the caudal brainstem in rats demonstrated using intracellular biotinamide. J Comp Neurol 358: 63-78, 1995.

18. Luo P, Wong R, Dessem D. Ultrastructural basis for synaptic transmission between jaw-muscle spindle afferents and trigeminothalamic neurons in the rostral trigeminal sensory nuclei of the rat. J Comp Neurol 363: 109-128, 1995.

19. Kang Y, Saito M, Hirai T. Masticatory movement and brain function. Dental Outlook 102: 406-416, 2003.

20. Shephard R J. Control mechanisms: Neuromuscular system. In. Physiology and biochemistry of exercise. 245-289, New York: CBS, 1982.

21. Gahery Y, Massion J. Co-ordination between posture and movement. Trends Neurosci 4: 199-202, 1981. 